\title{
Estimating and Localizing External Forces Applied on Flexible Instruments by Shape Sensing
}

\author{
Qiao Qiao $^{1,2}$, Dries Willems ${ }^{1}$, Gianni Borghesan ${ }^{1,2}$, Mouloud Ourak ${ }^{1}$, Joris De Schutter ${ }^{1,2}$ \\ and Emmanuel Vander Poorten ${ }^{1}$
}

\begin{abstract}
Force sensing is highly desirable in medical applications showing great potential to reduce tissue damage and enhance manipulation safety. Embedding force sensors in medical devices is however challenging and costly. Provided that the medical device is long, slender and largely compliant, we show in this paper that it is possible to estimate multiple loads along the length of the flexible instrument using a Cosserat-rod model and Fiber Bragg Grating sensors, which allows simultaneous shape and force sensing. We perform experiments that illustrate the feasibility of using this method to locate multiple contact forces along the instrument, and to estimate the magnitudes of the forces that are applied along the length of the instrument. Results show that one-directional force magnitude and location can be estimated with an average error of $6.3 \%$ and $5.4 \mathrm{~mm}$ (3.0\% of rod length), respectively. In the worst case, the error for magnitude and location can go up to $22.6 \%$ and $13.9 \mathrm{~mm}$ ( $7.7 \%$ of rod length), respectively. For two-directional forces, results show an average error of $9.8 \%$ for magnitude and $6.4 \mathrm{~mm}(3.6 \%$ of rod length) for the location. The errors on magnitude and location do not exceed $27.4 \%$ and $17.4 \mathrm{~mm}$ $(9.7 \%$ of rod length), respectively.
\end{abstract}

\section{INTRODUCTION}

In medical robotics, continuum robots have enjoyed increased interest due to their dexterous manipulation and intrinsic safety in cluttered and constrained environments. These robots can be easily miniaturized and can reach locations in the human body that are inaccessible when using rigid robots, making continuum robots appealing for Minimally Invasive Surgery (MIS). Despite this promise, it remains a challenging task to measure the interaction forces that can occur along the length of the continuum robot, between the instrument and its environment, which is key to ensure a safe operation within the often fragile environment.

Miniature force sensors placed at the tip of surgical tools have been presented in [1-4]. However, aspects such as miniaturization, cost, robustness and sterilizability limit their use. More importantly, these force sensors can only sense local forces at the tip and are blind to forces that arise along the length of the robot. End-effector force estimation schemes based on known actuation loads have been proposed for multi-backbone [5] and parallel [6] continuum robots. Besides actuation-based wrench sensing, also the compliant nature of the continuum robots itself could be explored to estimate forces through so-called deflection-based wrench sensing schemes. In [7], the tip contact force was estimated based on the pose of the catheter tip by modelling the catheter as a planar elastica. In [8], the magnitude and

\footnotetext{
${ }^{1}$ Department of Mechanical Engineering, KU Leuven, Belgium

${ }^{2}$ Core Lab ROB of Flanders Make, Belgium
}

direction of the tip contact force was calculated by a multisection kinematic model based on an Euler-Bernoulli representation. The tip contact force of a steerable catheter was estimated in [9] by studying the change in the catheter shape under different loading scenarios. Back et al. estimated the tip force using a Cosserat rod model based on the measured robot shape [10]. Rucker and Webster proposed a probabilistic approach based on an Extended Kalman Filter (EKF) to estimate the tip force, also here using measurements of the tip pose [11]. Another method to estimate external forces is to sense the strains on the robot directly using Fiber Bragg Grating sensors [12-13]. Nonetheless, most force sensing efforts, be it actuation-, deflection- or strain-based, have focused on sensing of a single force at the tip of the continuum robot, while estimation of external forces along the length of the robot remains a major challenge. The exception is the work from Bajo et al. who investigated the force contact locations along the shaft of the manipulator [1415]. However, these works cannot simultaneously provide the magnitude and the location of the external force.

In this paper, contact forces applied at arbitrary locations along the continuum robot are estimated using strain measurements from distributed multi-core FBG sensors. Such FBG sensors are also capable of providing shape information. This work shows how they can be exploited for simultaneous shape and force sensing. This paper extends the work in [12] where only tip forces were estimated. Both magnitude and location of the external forces applied along the continuum robot are estimated in this paper. Besides, the method proposed here is capable of estimating simultaneously multiple external forces applied in different directions. In this study, a Cosserat rod model is employed in conjunction with the FBG sensor measurements. Results for one and two loads are presented to showcase the proposed approach.

The remainder of the paper is arranged as follows. Section II describes a shape reconstruction algorithm from distributed multi-core Fiber Bragg Grating sensing. The force sensing method is detailed in Section III. The method is developed with the assumption of a large compliancy and multiple external loads to be applied, but distributed force and dynamics are not considered at this stage. Section IV covers the experimental results of the proposed force sensing methods. Finally, Section V concludes the paper and provides directions for future work. 

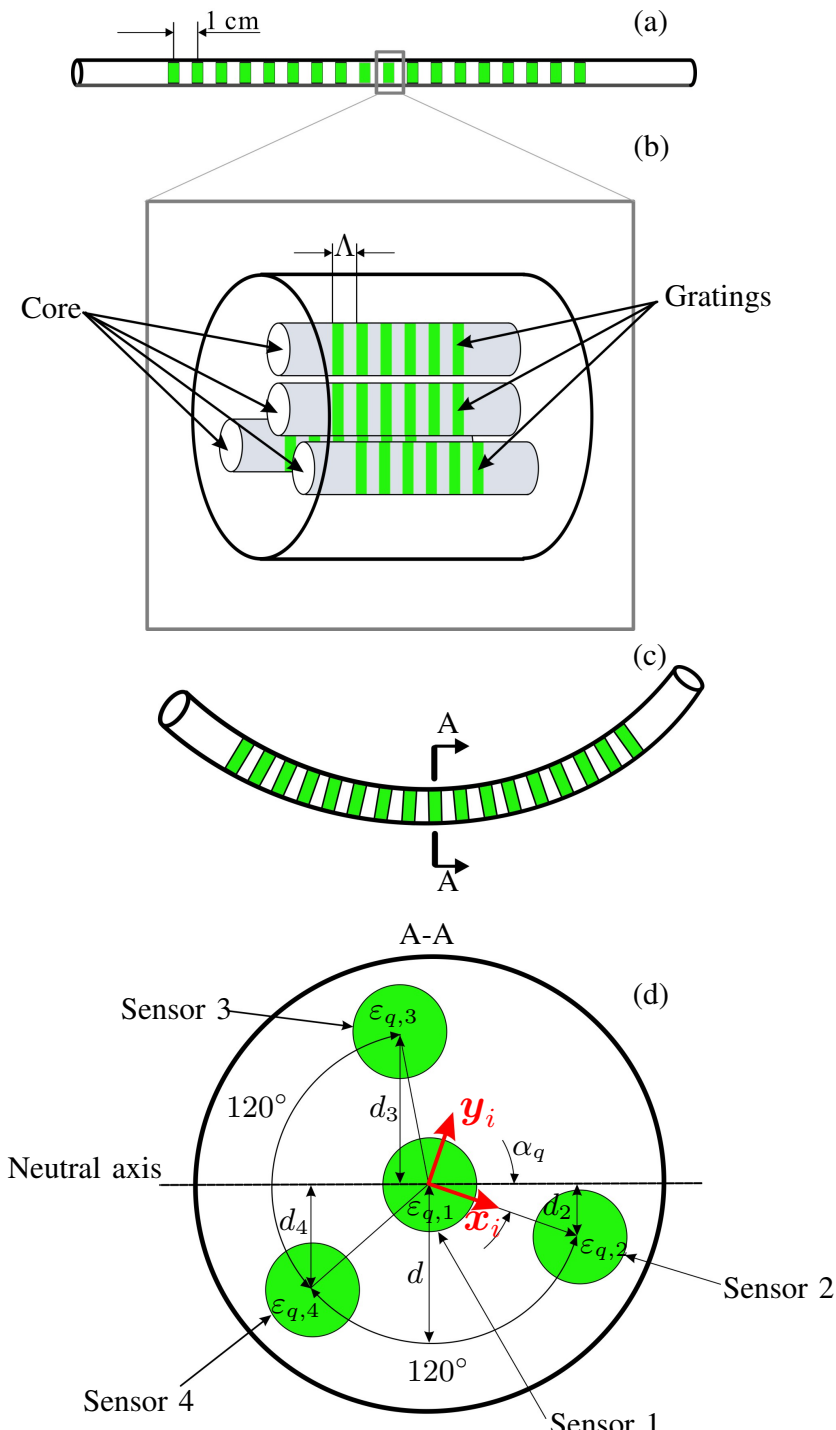

Fig. 1. A multicore fiber bragg grating sensor with 4 cores and 18 sets of gratings per core. (a) straight section containing 18 sets of gratings ( $q=$ $1: 18$ ) per core; (b) zoom in on a single set of gratings for the 4 cores (c) fiber in bent configuration; (d) cross-section at grating set " $q$ " showing the relative position of the three satellite cores $(2,3,4)$ and the central core (1).

\section{SHAPE RECONSTRUCTION}

This section introduces the principle of Fiber Bragg Grating sensors, which provide strain measurements that can be used for both shape reconstruction and force sensing. A method for the latter is introduced in Section III.

\section{A. Fiber Bragg Grating Sensors}

Fig. 1 shows the Fiber Bragg Grating sensor consisting of four cores with 18 gratings evenly distributed along each core. The distance between two sets of gratings is $1 \mathrm{~cm}$. Each grating reflects one particular wavelength (the Bragg wavelength $\lambda_{B}$ ) while transmitting all other wavelengths when broadband light is sent into the fiber. Both temperature change $\Delta T$ and mechanical strain $\varepsilon$ influence the Bragg wavelength. The measured wavelength shift $\Delta \lambda$ is given by

$$
\Delta \lambda=\lambda_{B}\left(\left(1-P_{\varepsilon}\right) \varepsilon+\left(\alpha_{\Lambda}+\alpha_{n}\right) \Delta T\right),
$$

where $P_{\varepsilon}$ is the photo-elastic coefficient of the fiber, and $\alpha_{\Lambda}$ and $\alpha_{n}$ are the thermo expansion coefficient and the thermo-optic coefficient, respectively. A typical value of 0.22 is selected for $P_{\varepsilon}$ in this paper. The reference wavelength $\lambda_{B}$ is measured when the fiber is in the straight configuration. Each grating serves as a strain gauge. With the four-core and 18 gratings per core, distributed 3D shape sensing is possible over a distance of $18 \mathrm{~cm}$.

\section{B. Curvature Calculation}

At each cross section where the sensor is located, the four-core fiber provides four strain values which can be used to calculate the direction and magnitude of the threedimensional bending. The relationship between the strain $\varepsilon_{q, j}$ measured by each core $(j=1: 4)$ at a particular sensor location $q$ along the fiber, the maximal bending strain $\varepsilon_{q, \max }$ and the corresponding bending direction $\alpha_{q}$ at the same location is

$$
\begin{gathered}
\varepsilon_{q, 2}=\varepsilon_{q, 1}-\kappa_{q} \cdot d_{2}=\varepsilon_{q, 1}-\sin \left(\alpha_{q}\right) \cdot \varepsilon_{q, \max } \\
\varepsilon_{q, 3}=\varepsilon_{q, 1}-\kappa_{q} \cdot d_{3}=\varepsilon_{q, 1}-\sin \left(\alpha_{q}+120^{\circ}\right) \cdot \varepsilon_{q, \max } \\
\varepsilon_{q, 4}=\varepsilon_{q, 1}-\kappa_{q} \cdot d_{4}=\varepsilon_{q, 1}-\sin \left(\alpha_{q}+240^{\circ}\right) \cdot \varepsilon_{q, \max }
\end{gathered}
$$

where $\varepsilon_{q, j}(j=1: 4)$ is calculated by

$$
\varepsilon_{q, j}=\frac{1}{\lambda_{B}\left(1-P_{\varepsilon}\right)} \Delta \lambda
$$

The strain measured by the central core $\varepsilon_{q, 1}$ contains axial strain and temperature information and thus is used for compensation of these factors. The values $\varepsilon_{q, 2}, \varepsilon_{q, 3}$ and $\varepsilon_{q, 4}$ are the strains measured by the outer cores which are spaced over 120 degrees with respect to the central core. The variable $d_{j}$ is the distance of core $j$ to the neutral axis of the fiber. The system of equations (2)-(4) is solved using iterative least square method. The curvature $\kappa_{q}$ can be calculated from the maximum bending strain $\varepsilon_{q, \max }$ by

$$
\kappa_{q}=\frac{\varepsilon_{q, \max }}{d},
$$

where $d$ is the distance from the outer to the central core.

Combining the magnitude of curvature $\kappa_{q}$ and bending direction $\alpha_{q}$, the curvature vector ${ }_{i} \boldsymbol{u}_{q}$ at each sensor location can be constructed as

$$
{ }_{i} \boldsymbol{u}_{q}=\left[\begin{array}{l}
u_{q, x} \\
u_{q, y} \\
u_{q, z}
\end{array}\right]=\left[\begin{array}{c}
\kappa_{q} \sin \left(\alpha_{q}\right) \\
\kappa_{q} \cos \left(\alpha_{q}\right) \\
\operatorname{tors}
\end{array}\right],
$$

where the left subscript $i$ indicates that the curvature vector ${ }_{i} \boldsymbol{u}_{q}$ at the location $q$ is expressed in the local or the body frame $\{i\}$. The body frame is attached to each point along the center line of the fiber, with its $x_{i}$ axis pointing to core 2 and $z_{i}$ axis being normal to the cross section. Finally, the $y_{i}$ axis is chosen such that a right-handed coordinate system is formed. 


\section{3D Shape Reconstruction}

To obtain the continuous curvature vector ${ }_{i} \boldsymbol{u}$, spline interpolation of the discrete ${ }_{i} \boldsymbol{u}_{q}$ is performed. After that, multiple methods can be employed to reconstruct the $3 \mathrm{D}$ shape from the continuous curvature vector. In [16], the shape is obtained by integrating the curvature vector ${ }_{i} \boldsymbol{u}$ twice. However, this approach is only applicable to small deflections as the integration is performed for the $x$ component of the arc length $s$ instead of the arc length $s$ itself. In [12] and [17], integration of the tangent vector is employed to reconstruct the shape. Using this method, the orientation of the tangent vector has to be determined first using the curvature vector ${ }_{i} \boldsymbol{u}$ before integrating. A more convenient and general method to obtain the shape is based on the integration of the rod kinematics which is shown as

$$
\begin{gathered}
\frac{d_{w} \boldsymbol{p}}{d s}={ }_{w} \boldsymbol{R}_{i} \boldsymbol{v} \\
\frac{d_{w} \boldsymbol{R}}{d s}={ }_{w} \boldsymbol{R}_{i} \hat{\boldsymbol{u}},
\end{gathered}
$$

where ${ }_{w} \boldsymbol{p}$ and ${ }_{w} \boldsymbol{R}$ are the position and orientation of the body frame $\{i\}$ with respect to the world frame $\{w\}$, respectively. ${ }_{i} \boldsymbol{v}$ is the rate of change of ${ }_{w} \boldsymbol{p}$ with respect to $s$ expressed in the body frame $\{i\}$. It is also known as the linear strain which represents the extension or contraction of the robot. For the robot employed in this paper, the linear strain ${ }_{i} \boldsymbol{v}$ is $[0,0,1]^{T}$ [18]. The ${ }^{\wedge}$ represents the skew symmetric matrix form of the vector variable.

\section{FORCE SENSING METHOD}

The Cosserat rod model is employed to link between the strain information obtained from the FBG sensors and the force estimation.

\section{A. Cosserat Rod Model}

The equilibrium differential equations of the Cosserat rod model describe the rate of change of the internal moment vector $\boldsymbol{m}(s)$ and the internal force vector $\boldsymbol{n}(s)$ expressed in the world frame $\{w\}$ with respect to the arc length as follows:

$$
\begin{gathered}
\frac{d_{w} \boldsymbol{m}}{d s}=-\frac{d_{w} \boldsymbol{p}}{d s} \times{ }_{w} \boldsymbol{n}-{ }_{w} \boldsymbol{l} \\
\frac{d_{w} \boldsymbol{n}}{d s}=-{ }_{w} \boldsymbol{f}
\end{gathered}
$$

where ${ }_{w} \boldsymbol{l}$ and ${ }_{w} \boldsymbol{f}$ are the distributed moment and force applied along the rod length. In this paper, we assume that there is no distributed load applied to the robot. To simplify the calculations later, the above equations are transformed to the body frame $\{i\}$ by employing the rotation matrix ${ }_{w} \boldsymbol{R}^{-1}$ from world frame $\{w\}$ to body frame $\{i\}$ :

expressed in the body frame $\{i\}$ :

$$
\begin{gathered}
\frac{d_{i} \boldsymbol{m}}{d s}=-{ }_{i} \hat{\boldsymbol{u}} \cdot{ }_{i} \boldsymbol{m}-{ }_{i} \hat{\boldsymbol{v}} \cdot{ }_{i} \boldsymbol{n}-{ }_{i} \boldsymbol{l} \\
\frac{d_{i} \boldsymbol{n}}{d s}=-{ }_{i} \hat{\boldsymbol{u}} \cdot{ }_{i} \boldsymbol{n}-{ }_{i} \boldsymbol{f} .
\end{gathered}
$$

The internal moment and force are related to ${ }_{i} \boldsymbol{u}$ and ${ }_{i} \boldsymbol{v}$ via the constitutive law. For the static application in this paper, a linear law is employed:

$$
\begin{gathered}
{ }_{i} \boldsymbol{u}=\boldsymbol{K}_{u}{ }^{-1} \cdot{ }_{i} \boldsymbol{m}+{ }_{i} \boldsymbol{u}_{0} \\
{ }_{i} \boldsymbol{v}=\boldsymbol{K}_{v}{ }^{-1} \cdot{ }_{i} \boldsymbol{n}+{ }_{i} \boldsymbol{v}_{0}
\end{gathered}
$$

where

$$
\begin{gathered}
\boldsymbol{K}_{u}(s)=\operatorname{diag}\left(E(s) I_{x x}(s), E(s) I_{y y}(s), G(s) I_{z z}(s)\right) \\
\boldsymbol{K}_{v}(s)=\operatorname{diag}(G(s) A(s), G(s) A(s), E(s) A(s))
\end{gathered}
$$

$\boldsymbol{K}_{u}(s)$ is the matrix for bending and torsional stiffness and $\boldsymbol{K}_{v}(s)$ describes the shear and axial stiffness. The above matrixes include the area of the cross section $A(s)$, the Young's modulus $E(s)$, the shear modulus $G(s)$, the second area moment $I_{x x}$ and $I_{y y}$, and the polar area moment $I_{z z}$. The kinematic variables ${ }_{i} \boldsymbol{u}_{0}$ and ${ }_{i} \boldsymbol{v}_{0}$ describe the initial unloaded configuration of the continuum robot. For an initially straight configuration, the reference state variables are ${ }_{i} \boldsymbol{u}_{0}=[0,0,0]^{T}$ and ${ }_{i} \boldsymbol{v}_{0}=[0,0,1]^{T}$.

\section{B. Force Sensing Method}

After obtaining the curvature vector ${ }_{i} \boldsymbol{u}$ from the shape sensing section, the internal moment ${ }_{i} \boldsymbol{m}$ can be readily calculated using (14) from the constitutive law. In this paper, it is assumed that only external forces are applied on the robot. As a result, the internal moment ${ }_{i} \boldsymbol{m}$ varies in a continuous manner.

In order to estimate the magnitude of the external force, the most straightforward method is to take the derivative of the internal moment according to (12). Then one can make use of: ${ }_{i} \boldsymbol{n}\left(s_{E, j}^{+}\right)={ }_{i} \boldsymbol{n}\left(s_{E, j}^{-}\right)-{ }_{i} \boldsymbol{F}_{E, j}$, which states that at the single point contact location $j$ (for $j=1,2, \ldots, N$ ), the magnitudes of the external forces ${ }_{i} \boldsymbol{F}_{E, j}$ at contact location $s_{E, j}$ can be found as the difference between the internal force ${ }_{i} \boldsymbol{n}\left(s_{E, j}^{-}\right)$at the location just before the contact location $s_{E, j}^{-}$ and the internal force ${ }_{i} \boldsymbol{n}\left(s_{E, j}^{+}\right)$at $s_{E, j}^{+}$just after the contact location. However, due to measurement noise and the use of a derivative action, one may observe unexpected and incorrect external forces if applying the method directly. To eliminate these artefacts, we propose to make use of prior knowledge.

Since the ideal internal moment is composed of piecewise straight lines when only local external forces are applied along the robot, piecewise lines can be fitted through the data to estimate the location of force application which is the intersection of well fitting lines. A Top-Down segmentation method is employed in this paper:

- The algorithm starts by assuming every data point can be the breakpoint.

- The data point with the smallest fitting error will be the actual breakpoint.

- The data set is broken into two segments. The algorithm will be called again for either segment which does not meet the stopping criteria.

- The process contnues until a stopping criteria is met for each segment. 
The stopping criterion is determined by multiplying the data by the strain variation over all 18 sensors of the fiber (0.05). The criterion checks for each segment if the L2norm of the residuals to the fit is smaller than the L2-norm of the variation for that segment. After the segmentation, least squares line fitting is performed for each segment. The adjacent segments are extended until they intersect with each other. The locations where external forces are applied are detected by searching the location where lines intersect. This corresponds to locations where the slope of the internal moment changes. Employing this technique, also the contact locations between adjacent gratings can be detected, thus theoretically leading to a resolution better than the spacing between two adjacent gratings, i.e. lower than $1 \mathrm{~cm}$. The internal force can be calculated from (12) using the linear segmented internal moment and the magnitudes of the external forces can be obtained from ${ }_{i} \boldsymbol{n}\left(s_{E, j}^{+}\right)={ }_{i} \boldsymbol{n}\left(s_{E, j}^{-}\right)-{ }_{i} \boldsymbol{F}_{E, j}$ at each location that was detected in the previous step.

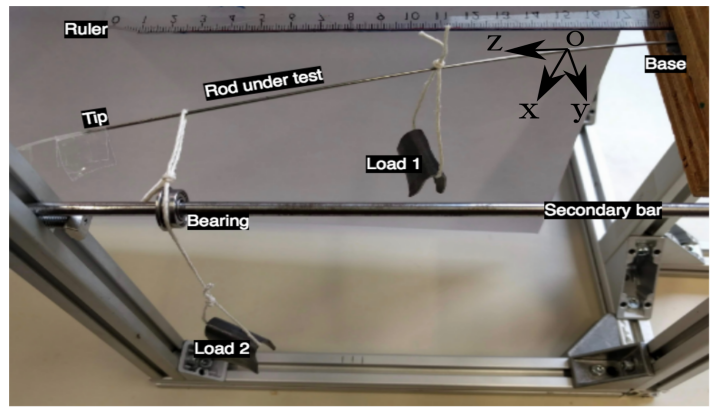

(a)

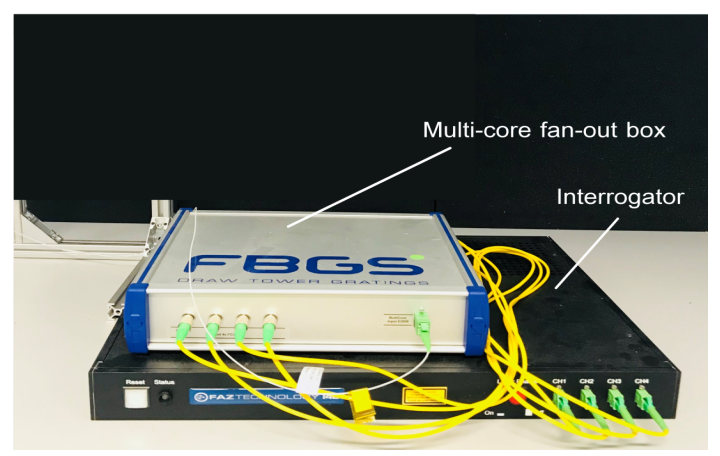

(b)

Fig. 2. Experimental setup: (a) elastic rod with two-directional forces applied along its length, and (b) FBG multi-core fan-out box and interrogator.

\section{EXPERIMENTAL VALIDATION}

To test our proposed force sensing method, we performed a series of experiments applying forces both in one and in two different directions.

\section{A. Experimental Setup}

The experimental setup that was used is shown in Fig. 2 (a). It consists of an elastic rod with its base clamped rigidly. A weight is suspended to apply a vertical load. A secondary bar with a bearing is used to apply a second load. This force is applied in the direction perpendicular to
TABLE I

ERROR ON THE EXPERIMENTS OF ONE-DIRECTIONAL LOAD LOCALIZATION. A LOAD OF $0.113 \mathrm{~N}$ IS APPLIED EVERY $0.33 \mathrm{CM}$ ALONG THE ROD.

\begin{tabular}{|ccc||ccc|}
\hline $\begin{array}{c}\text { True } \\
\text { location } \\
(\mathbf{m m})\end{array}$ & $\begin{array}{c}\text { Estimated } \\
\text { location } \\
(\mathbf{m m})\end{array}$ & $\begin{array}{c}\text { Error } \\
\text { in } \\
\text { location } \\
\mathbf{( m m}\end{array}$ & $\begin{array}{c}\text { True } \\
\text { location } \\
(\mathbf{m m})\end{array}$ & $\begin{array}{c}\text { Estimated } \\
\text { location } \\
(\mathbf{m m})\end{array}$ & $\begin{array}{c}\text { Error } \\
\text { in } \\
\text { location } \\
\mathbf{( m m})\end{array}$ \\
\hline 180.0 & 180.0 & 0.0 & 100.0 & 108.1 & -8.1 \\
\hline 176.6 & 180.0 & -3.4 & 96.6 & 103.0 & -6.4 \\
\hline 173.3 & 180.0 & -6.7 & 93.3 & 98.1 & -4.8 \\
\hline 170.0 & 180.0 & -10.0 & 90.0 & 94.3 & -4.3 \\
\hline 166.6 & 180.0 & -13.4 & 86.6 & 90.0 & -3.4 \\
\hline 163.3 & 165.5 & -2.2 & 83.3 & 89.4 & -6.1 \\
\hline 160.0 & 160.8 & -0.8 & 80.0 & 83.9 & -3.9 \\
\hline 156.6 & 157.7 & -1.1 & 76.6 & 78.3 & -1.7 \\
\hline 153.3 & 153.0 & 0.3 & 73.3 & 78.3 & -5.0 \\
\hline 150.0 & 150.3 & -0.3 & 70.0 & 74.5 & -4.5 \\
\hline 146.6 & 149.1 & -2.5 & 66.6 & 76.1 & -9.5 \\
\hline 143.3 & 147.6 & -4.3 & 63.3 & 72.5 & -9.2 \\
\hline 140.0 & 145.2 & -5.2 & 60.0 & 65.7 & -5.7 \\
\hline 136.6 & 130.9 & 5.7 & 56.6 & 65.6 & -9.0 \\
\hline 133.3 & 135.4 & -2.1 & 53.3 & 57.0 & -3.7 \\
\hline 130.0 & 137.3 & -7.3 & 50.0 & 56.5 & -6.5 \\
\hline 126.6 & 133.9 & -7.3 & 46.6 & 52.0 & -5.4 \\
\hline 123.3 & 127.6 & -4.3 & 43.3 & 50.0 & -6.7 \\
\hline 120.0 & 123.5 & -3.5 & 40.0 & 37.4 & 2.6 \\
\hline 116.6 & 124.7 & -8.1 & 36.6 & 46.0 & -9.4 \\
\hline 113.3 & 121.7 & -8.4 & 33.3 & 29.0 & 4.3 \\
\hline 110.0 & 114.9 & -4.9 & 30.0 & 31.8 & -1.8 \\
\hline 106.6 & 113.3 & -6.7 & 26.6 & 32.0 & -5.4 \\
\hline 103.3 & 112.3 & -9.0 & 23.3 & 9.4 & 13.9 \\
\hline & & & & & \\
\hline
\end{tabular}

TABLE II

ERROR ON LOCATION ESTIMATION FOR A LOAD OF $0.0318 \mathrm{~N}$ IN X-DIRECTION AND A LOAD OF $0.113 \mathrm{~N}$ IN Y-DIRECTION.

\begin{tabular}{|c|c|c|c|c|c|c|c|c|c|}
\hline & \multicolumn{2}{|c|}{$0.0318 \mathrm{~N}$} & \multicolumn{2}{|c|}{$0.113 \mathrm{~N}$} & & \multicolumn{2}{|c|}{$0.0318 \mathrm{~N}$} & \multicolumn{2}{|c|}{$0.113 N$} \\
\hline Case & $\begin{array}{l}\text { True } \\
\text { value } \\
\text { (mm) }\end{array}$ & $\begin{array}{l}\text { Error } \\
(\mathbf{m m})\end{array}$ & $\begin{array}{l}\text { True } \\
\text { value } \\
\text { (mm) }\end{array}$ & $\begin{array}{l}\text { Error } \\
(\mathbf{m m})\end{array}$ & Case & $\begin{array}{l}\text { True } \\
\text { value } \\
\text { (mm) }\end{array}$ & $\begin{array}{l}\text { Error } \\
(\mathrm{mm})\end{array}$ & $\begin{array}{l}\text { True } \\
\text { value } \\
\text { (mm) }\end{array}$ & $\begin{array}{l}\text { Error } \\
(\mathbf{m m})\end{array}$ \\
\hline 1 & 10 & 10.0 & 180 & 0 & 10 & 100 & -2.4 & 90 & -5.3 \\
\hline 2 & 20 & 20.0 & 170 & 10.0 & 11 & 110 & -6.5 & 80 & 10.4 \\
\hline 3 & 30 & 6.6 & 160 & 20.0 & 12 & 120 & 1.6 & 70 & 10.3 \\
\hline 4 & 40 & 40.0 & 150 & -9.3 & 13 & 130 & 6.3 & 60 & 17.4 \\
\hline 5 & 50 & 8.8 & 140 & -3.7 & 14 & 140 & 9.7 & 50 & 16.1 \\
\hline 6 & 60 & -2.3 & 130 & 7.6 & 15 & 150 & 10.8 & 40 & 11.2 \\
\hline 7 & 70 & -0.2 & 120 & 7.2 & 16 & 160 & 11.1 & 30 & 12.8 \\
\hline 8 & 80 & 8.0 & 110 & 4.2 & 17 & 170 & 10.0 & 20 & 20.0 \\
\hline 9 & 90 & 0.1 & 100 & 0.7 & 18 & 180 & 0 & 10 & 10.0 \\
\hline
\end{tabular}

that of gravity. The rod equipped with the FBG fiber in the experiment is made from stainless steel with a length of 180 $\mathrm{mm}$, an outer diameter of $1.2 \mathrm{~mm}$ and an inner diameter of $1 \mathrm{~mm}$. The Young's modulus of the rod is $195 \mathrm{GPa}$ and the shear modulus is $74 \mathrm{GPa}$. The shape sensor, which has the diameter of $0.195 \mathrm{~mm}$ and outer-core-to-central-core distance of $3.65 \times 10^{-5} \mathrm{~m}$, is inserted in the center of the hollow rod. The fiber is manually registered such that the origin of the fiber aligns with the base of the rod. As the rod bends due to external loads, also the shape sensor will bend.

As shown in Fig. 2 (b), the FBG sensors are connected to an interrogator (FAZT I4E, FAZ Technology Inc., Ireland) 


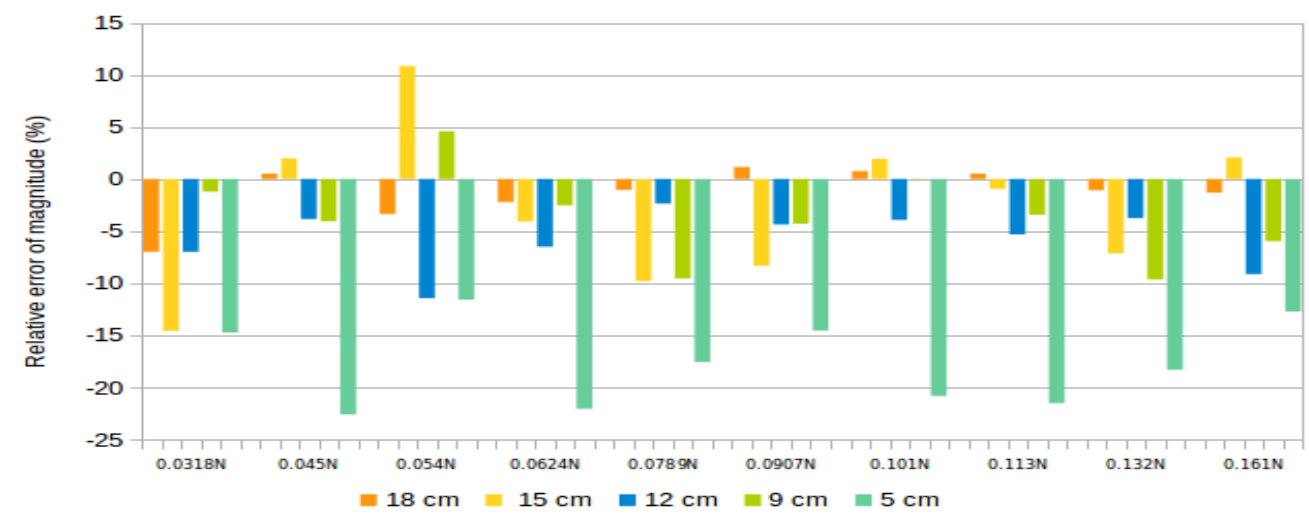

(a)

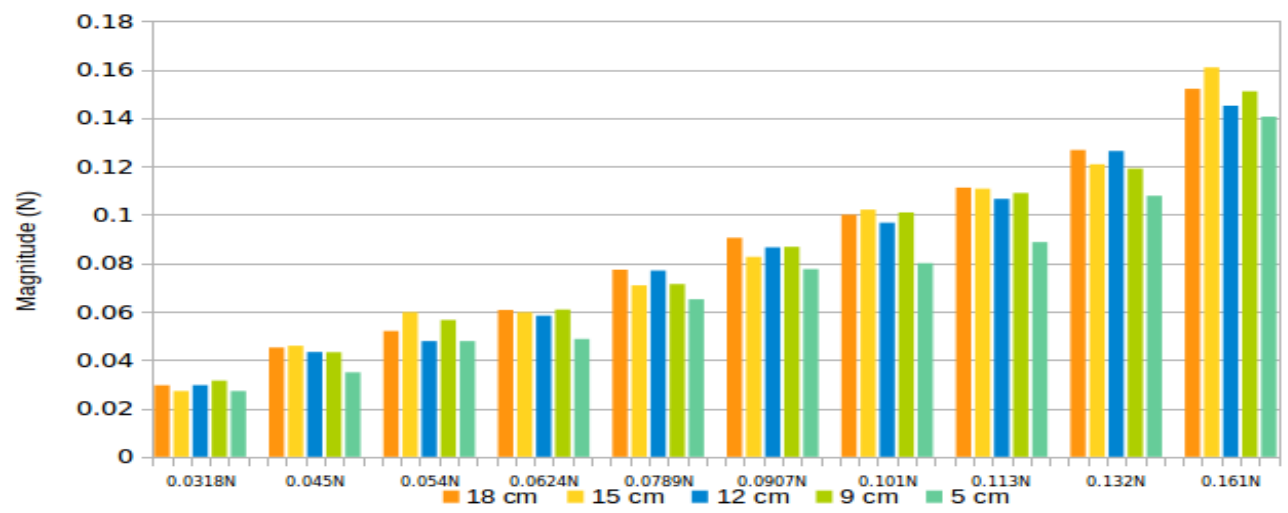

(b)

Fig. 3. Accuracy of one-directional load magnitude estimation. 10 different loads are applied at 5 locations along the rod: (a) relative magnitude error and (b) estimated magnitude.

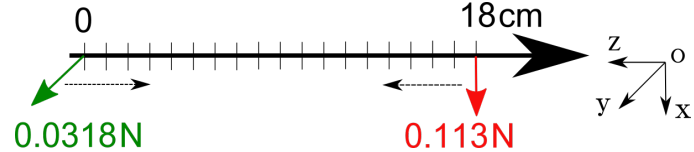

Fig. 4. Illustration of the loading case for forces applied in two directions.

through a multi-core fan-out box (FBGS, Belgium). The interrogator measures the reflected wavelengths of each FBG core in parallel. The shape sensing fiber is a multi-core fiber with Draw Tower Gratings (DTG) from FBGS. All $4 \times 18$ sensor data are acquired at a rate of $500 \mathrm{~Hz}$. The data from the FBG fiber is sent over Ethernet to a personal computer. To characterize errors of the FBG fiber, a calibration process is performed in the experiment by employing planar slots with known constant curvature.

The rod is bent to different shapes by suspending weights at different locations along the rod's length. The places where the loads are applied are recorded. These form the groundtruth locations. Since the rod bends, the applied load will split out in a component that is perpendicular to the rod and a component that is aligned with the longitudinal rod axis. The Cosserat rod model expressed in the world frame is solved from the tip to the base using ode45, and thus the rotation matrix from the body frame where the load is applied to the base frame is obtained. The forces created by the loads in the experiment which are specified in the world frame can be transformed to the body frame where the load is applied to obtain the perpendicular component of the load. This forms a reference for the ground-truth magnitude of the load.

\section{B. Results}

In the calibration process, a general trend of underestimation in $\varepsilon_{\max }$ by $2 \%$ can be recognized. The 18 gratings independently have an offset between $0 \%$ and $-9 \%$ of the true strain. The bending direction $\alpha$ is measurable with an absolute error of 0.085 rad for bending in a plane.

1) One-Directional Force Localization: In the first set of experiments, only one load was applied at varying locations along the rod. To test the accuracy of the estimation of the magnitude of the applied force, 10 different loads ranging from $0.0318 \mathrm{~N}$ to $0.161 \mathrm{~N}$ are applied once at 5 different locations, namely at $5 \mathrm{~cm}, 9 \mathrm{~cm}, 12 \mathrm{~cm}, 15 \mathrm{~cm}$ and $18 \mathrm{~cm}$ (tip) from the base. Fig. 3 shows the result of the magnitude estimations and relative errors. The true load is in general underestimated. The average error is $6.3 \%$. In the worst case, the error goes up to $22.6 \%$. It can be seen that the accuracy is higher when the load is applied near the tip. To characterize the errors on localization, one load of $0.113 \mathrm{~N}$ is applied at 50 equally spaced locations along the rod. Table I shows the 


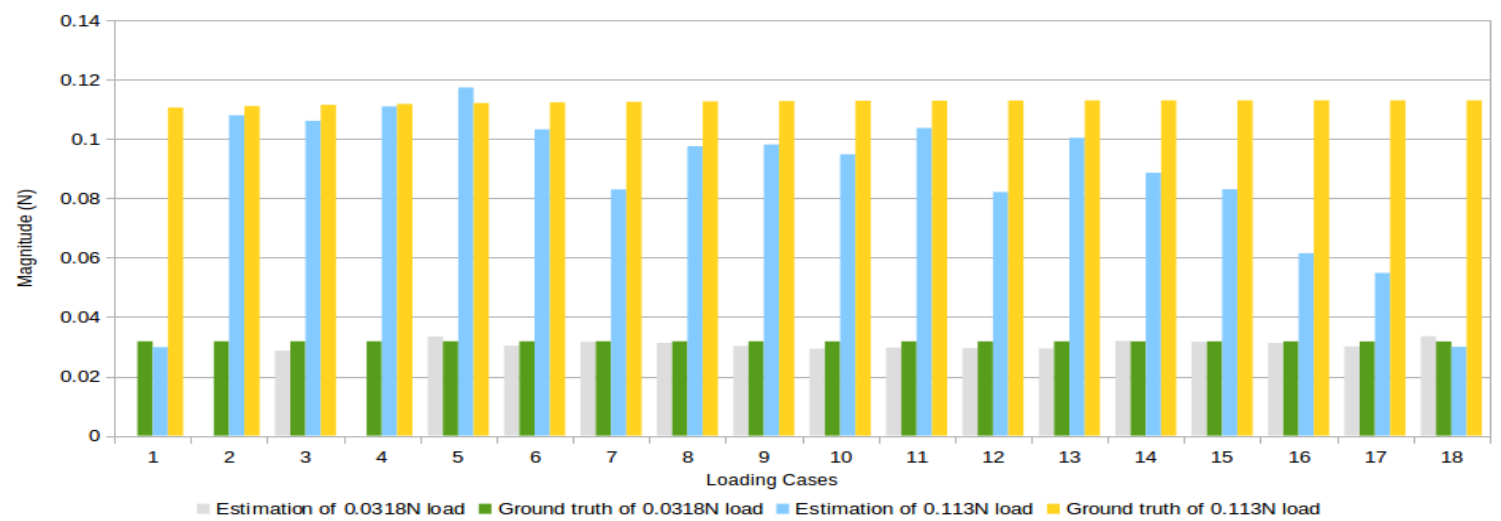

Fig. 5. Error on the magnitude estimation for a load of $0.0318 \mathrm{~N}$ in the $\mathrm{x}$-direction and a load of $0.113 \mathrm{~N}$ in the $\mathrm{y}$-direction.

error on the localization. On average, the error is $5.4 \mathrm{~mm}$. In the worst case, the error can go up to $13.9 \mathrm{~mm}$ when the load is applied close to the base.

Experiments are conducted to verify the repeatability of magnitude and location estimation. A $0.113 \mathrm{~N}$ load is applied 10 times at 5 locations along the rod. The results are represented by a boxplot to visualize the average, spreading and minimal/maximal values, as shown in Fig. 6. The brown boxplot shows the results when the load is re-applied 10 times at a certain location. The blue boxplot represents the case when the load is applied once, kept stationary and measured every minute during 10 minutes. The spreading in the latter is noticeably smaller than the spreading in the brown boxplots. Applying the load every time again leads to a spreading up to $0.8 \mathrm{~cm}$ on the location and $0.02 \mathrm{~N}$ for the load magnitude. Considering the measurements when the load is not re-applied, the spreading of the location ranges up to $0.15 \mathrm{~cm}$, while the spreading on the magnitude of the load is up to $0.008 \mathrm{~N}$. An explanation for this could be the slight variation of the location every time the load is re-applied.

2) Bi-Directional Force Localization: In a second set of experiments, a secondary bar with a bearing is used to generate forces perpendicular to the direction of the gravity. The experiments were conducted with a large $(0.113 \mathrm{~N})$ and a small $(0.0318 \mathrm{~N})$ load, applied in perpendicular directions. Fig. 4 illustrates the tested loading case. The red load of $0.113 \mathrm{~N}$ is applied in the direction of the gravity ( $\mathrm{X}$ direction). The green load of $0.0318 \mathrm{~N}$ is applied in the perpendicular direction ( $\mathrm{Y}$ direction). The two loads are moved along the axis of the rod, and the magnitude and location are measured every centimeter. Fig. 5 and Table II show the results on magnitude and location estimation, respectively. As can be seen from loading cases 1,2 and 4 in Fig. 5, the $0.0318 \mathrm{~N}$ load cannot be detected due to the limited deflection when it is applied near the base. It can also be seen that large estimation error occurs when the $0.113 \mathrm{~N}$ load is exerted near the base. Excluding the cases when the load is applied near the base, the results on the load magnitude show an average error of $9.8 \%$. The error on the true load does not exceed $27.4 \%$. The average error of the

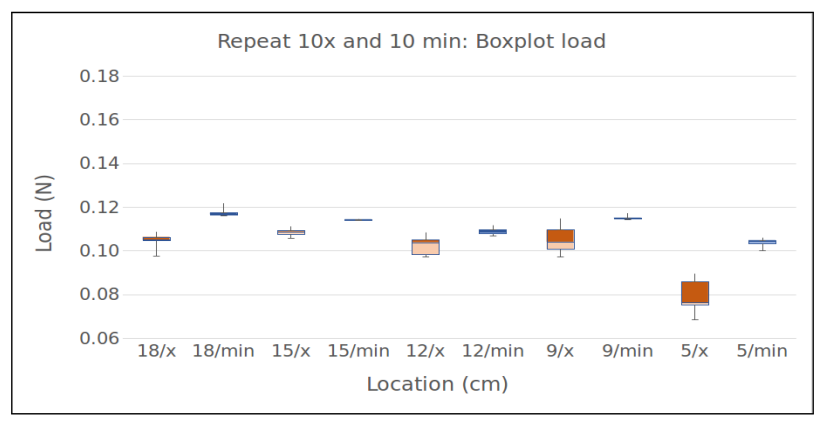

(a)

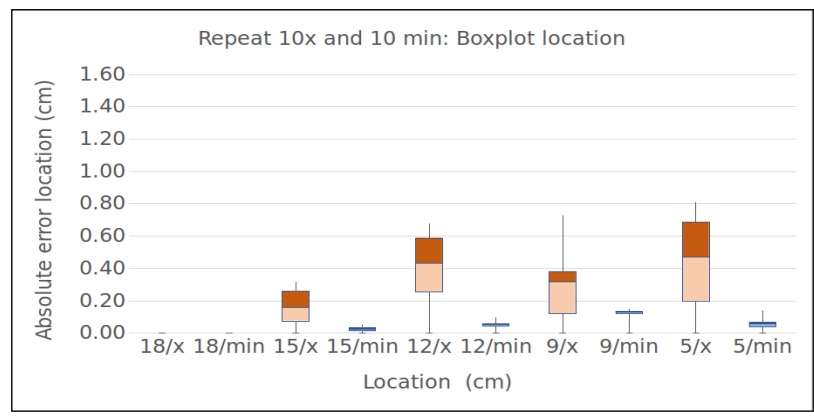

(b)

Fig. 6. Experiments on repeatability of one-directional force sensing: (a) shows the results on magnitude and (b) represents results on location.

location estimation is $6.4 \mathrm{~mm}$ and the error is always smaller than or equal to $17.4 \mathrm{~mm}$. A reason for the higher error in the y-direction can also be found in the experimental setup itself. The load in the y-direction is generated by suspending on a known mass over a bearing at the secondary rod. The frictional forces between the rope and the bearing surface can affect the load in such a way that the true load differs slightly from the suspended weight. Furthermore, the error decreases towards the middle of the rod $(9 \mathrm{~cm})$ as expected due to a smaller fitting error when the segments are fitted using more strain data.

To investigate the repeatability of the two-directional force, the load case of $0.113 \mathrm{~N}$ at $7 \mathrm{~cm}$ in the $\mathrm{x}$-plane and $0.113 \mathrm{~N}$ at $11 \mathrm{~cm}$ in the y-plane is measured 10 times. In Fig. 


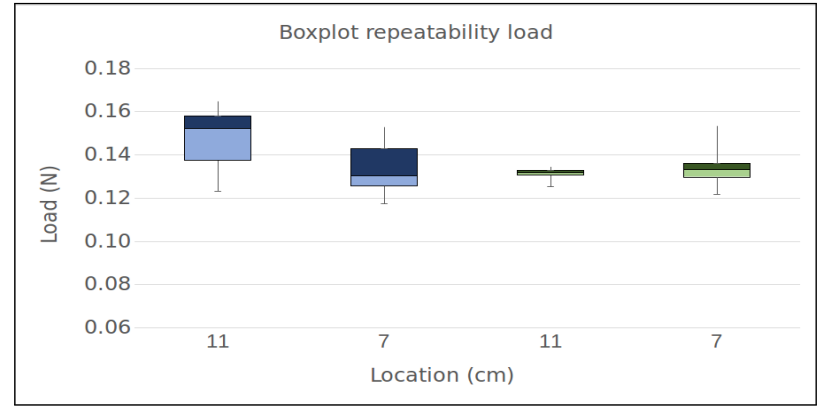

(a)

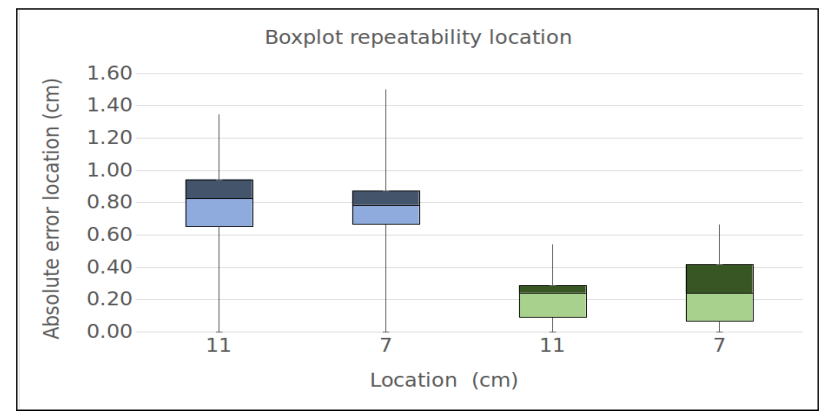

(b)

Fig. 7. Experiments on repeatability of two-directional force sensing: (a) shows the results on magnitude and (b) represents results on location.

7 , the blue plots show the results when the load is applied 10 times. The green plots show the variation if the load is applied once and measured every minute during 10 minutes. It can be seen that the spreading in both graphs is somewhat larger compared to the unidirectional measurements in Fig. 6. An explanation for this could be the variation of the bending direction $\alpha$. A slight change can cause here a large difference in the estimation results.

\section{CONCLUSIONS}

This paper presents a method that enables simultaneous shape and force sensing for continuum robots by employing distributed multi-core FBG sensors. The proposed force sensing method has the capability to yield useful information about the location and magnitudes of multiple forces on soft/continuum robots and flexible devices. Experimental results validated the feasibility of localization and magnitude estimation for both one and up to two forces in different directions. In future work, the bias of the FBG sensor observed during the calibration process can be compensated to improve the accuracy on magnitude and location estimation of the loads. In addition, the forces estimated using the method in this paper could be used in real-time for closed loop force control to improve manipulation accuracy and account for distributed contacts.

\section{ACKNOWLEDGMENT}

This work was partially funded by the Chinese Scholarship Council (CSC No. 201606290030) as well as by the European Union's Horizon 2020 research and innovation program under grant agreement No. 779813 (ATLAS) and two internal C2 KUL projects, i.e., SF-Reacta (3E180412) and PRESCLICE (3E160397). The authors would like to thank FBGS for their support in setting up the shape sensing hardware.

\section{REFERENCES}

[1] Shimachi, Shigeyuki, Yasunori Fujiwara, and Yoshihide Hakozaki. "New sensing method of force acting on instrument for laparoscopic robot surgery." International Congress Series. Vol. 1268. Elsevier, 2004

[2] Seibold, Ulrich, Bernhard Kubler, and Gerd Hirzinger. "Prototype of instrument for minimally invasive surgery with 6-axis force sensing capability." Proceedings of the 2005 IEEE International Conference on Robotics and Automation. IEEE, 2005.

[3] Pitakwatchara, Phongsaen, Shin-ichi Warisawa, and Mamoru Mitsuishi. "Force Feedback Augmentation Modes in the laparoscopic Minimal Invasive Telesurgical System." The First IEEE/RAS-EMBS International Conference on Biomedical Robotics and Biomechatronics, 2006.

[4] Valdastri, Pietro, et al. "Integration of a miniaturised triaxial force sensor in a minimally invasive surgical tool." IEEE transactions on biomedical engineering 53.11 (2006): 2397-2400.

[5] Xu, Kai, and Nabil Simaan. "Intrinsic wrench estimation and its performance index for multisegment continuum robots." IEEE Transactions on Robotics 26.3 (2010): 555-561.

[6] Black, Caroline B., John Till, and D. Caleb Rucker. "Parallel continuum robots: Modeling, analysis, and actuation-based force sensing." IEEE Transactions on Robotics 34.1 (2017): 29-47.

[7] Hasanzadeh, Shahir, and Farrokh Janabi-Sharifi. "Model-based force estimation for intracardiac catheters." IEEE/ASME Transactions on Mechatronics 21.1 (2015): 154-162.

[8] Back, Junghwan, et al. "New kinematic multi-section model for catheter contact force estimation and steering." 2016 IEEE/RSJ International Conference on Intelligent Robots and Systems (IROS), 2016.

[9] Khoshnam, Mahta, Allan C. Skanes, and Rajni V. Patel. "Modeling and estimation of tip contact force for steerable ablation catheters." IEEE Transactions on Biomedical Engineering 62.5 (2015): 1404-1415.

[10] Back, Junghwan, et al. "Catheter contact force estimation from shape detection using a real-time Cosserat rod model." IEEE/RSJ International Conference on Intelligent Robots and Systems (IROS), 2015.

[11] Rucker, D. Caleb, and Robert J. Webster. "Deflection-based force sensing for continuum robots: A probabilistic approach.” IEEE/RSJ International Conference on Intelligent Robots and Systems, 2011.

[12] Khan, Fouzia, Roy J. Roesthuis, and Sarthak Misra. "Force sensing in continuum manipulators using fiber bragg grating sensors." IEEE/RSJ International Conference on Intelligent Robots and Systems (IROS), 2017

[13] Xu, Ran, Aaron Yurkewich, and Rajni V. Patel. "Curvature, torsion, and force sensing in continuum robots using helically wrapped FBG sensors." IEEE Robotics and Automation Letters 1.2 (2016): 10521059

[14] Bajo, Andrea, and Nabil Simaan. "Finding lost wrenches: Using continuum robots for contact detection and estimation of contact location.” IEEE International Conference on Robotics and Automation, 2010.

[15] Bajo, Andrea, and Nabil Simaan. "Kinematics-based detection and localization of contacts along multisegment continuum robots." IEEE Transactions on Robotics 28.2 (2011): 291-302.

[16] Abayazid, Momen, Marco Kemp, and Sarthak Misra. "3d flexible needle steering in soft-tissue phantoms using fiber bragg grating sensors.” IEEE International Conference on Robotics and Automation, 2013

[17] Roesthuis, Roy J., and Sarthak Misra. "Steering of multisegment continuum manipulators using rigid-link modeling and FBG-based shape sensing." IEEE transactions on robotics 32.2 (2016): 372-382.

[18] Smoljkic, Gabrijel, et al. "Control of a hybrid robotic system for computer-assisted interventions in dynamic environments." International journal of computer assisted radiology and surgery 11.7 (2016): 1371-1383. 